OPEN ACCESS

Edited by:
Teresa Rolle,
University of Turin, Italy

Reviewed by:

Kun Xiong,

Central South University, China Gemma Caterina Maria Rossi,

San Matteo Hospital Foundation

(IRCCS), Italy

Weizhao Lu,

Shandong First Medical

University, China

*Correspondence:

Giorgia Demaria

g.demaria@umcg.n

tThese authors have contributed equally to this work

Specialty section:

This article was submitted to Neurocognitive Aging and Behavior,

a section of the journal

Frontiers in Aging Neuroscience

Received: 19 July 2021 Accepted: 20 December 2021

Published: 13 January 2022

Citation:

Demaria G, Invernizzi A, Ombelet $D$,

Carvalho JC, Renken RJ and

Cornelissen FW (2022) Binocular Integrated Visual Field Deficits Are Associated With Changes in Local

Network Function in Primary

Open-Angle Glaucoma: A

Resting-State fMRI Study.

Front. Aging Neurosci. 13:744139.

doi: 10.3389/fnagi.2021.744139

\section{Binocular Integrated Visual Field Deficits Are Associated With Changes in Local Network Function in Primary Open-Angle Glaucoma: A Resting-State fMRI Study}

\author{
Giorgia Demaria ${ }^{1 * t}$, Azzurra Invernizzi ${ }^{2 \dagger}$, Daniel Ombelet $^{2}$, Joana C. Carvalho ${ }^{2}$, \\ Remco J. Renken ${ }^{2}$ and Frans W. Cornelissen ${ }^{1,2}$
}

${ }^{1}$ Laboratory of Experimental Ophthalmology, University of Groningen, University Medical Center Groningen, Groningen, Netherlands, ${ }^{2}$ Cognitive Neuroscience Center, Department of Biomedical Sciences of Cells \& Systems, University Medical Center Groningen, Groningen, Netherlands

In glaucoma participants, both structural and functional brain changes have been observed, but we still have insufficient understanding of how these changes also affect the integrity of cortical functional networks, and how these changes relate to visual function. This is relevant, as functional network integrity may affect the applicability of future treatments, as well as the options for rehabilitation or training. Here, we compare global and local functional connectivity in local and global brain networks between glaucoma and control participants. Moreover, we study the relationship between functional connectivity and visual field (VF) loss. For our study, 20 subjects with primary open-angle glaucoma (POAG) and 24 age-similar healthy participants were recruited to undergo an ophthalmic assessment followed by two resting-state (RS) (f)MRI scans. For each scan and for each group, the ROls with eigenvector centrality (EC) values higher than the 95th percentile were considered the most central brain regions ("hubs"). Hubs for which we found a significant difference in EC in both scans between glaucoma and healthy participants were considered to provide evidence for network changes. In addition, we tested the notion that a brain region's hub function in POAG might relate to the severity of a participant's VF defect, irrespective of which eye contributed mostly to this. To determine this, for each participant, eye-independent scores were derived for: (1) sensitivity of the worse eye - indicating disease severity, (2) sensitivity of both eyes combined - with one eye potentially compensating for loss in the other, or (3) difference in eye sensitivity - potentially requiring additional network interactions. By correlating each of these VF scores and the EC values, we assessed whether VF defects could be associated with centrality alterations in POAG. Our results show that no functional connectivity disruptions were found at the global brain level in POAG participants. This indicates that in glaucoma global brain network communication is preserved. Furthermore, for the Lingual Gyrus, identified as a brain hub, we found a 
positive correlation between the EC value and the VF sensitivity of both eyes combined. The fact that reduced local network functioning is associated with reduced binocular VF sensitivity suggests the presence of local brain reorganization that has a bearing on functional visual abilities.

Keywords: visual field defects, HFA, resting-state fMRI, POAG, functional connectivity, eigenvector centrality, functional networks

\section{INTRODUCTION}

Glaucoma is a neurodegenerative ophthalmic disease, which is characterized, amongst others, by reduced retinal thickness and loss of visual field (VF) sensitivity. It is one of the leading causes of irreversible blindness, and in 2020 approximately 76 million people are affected by this disease, a number that is only estimated to increase in the coming years (Quigley and Broman, 2006; Tham et al., 2014). Thus, it is important to better understand the pathogenesis of glaucoma, in order to improve its diagnosis, prognosis and the possible treatment options, all of which will ultimately improve the quality of life of patients. While in the clinical setting the degenerative physical damage is generally observed in the eye and in the optic nerve, recent brain imaging studies have shown that this is not limited to these but extends intracranially along the visual pathways and into the visual cortex (Gupta and Yücel, 2007; Hernowo, 2012; Nucci et al., 2013; Haykal et al., 2021). It has been shown that structural and functional changes in the brain are involved in glaucoma. Structural changes were observed in several studies that compared glaucoma participants to healthy controls, showing altered white matter tracts and gray matter atrophy in brain areas involved in visual processing (Chen et al., 2013; Frezzotti et al., 2014; Wang et al., 2016a; Giorgio et al., 2018). Moreover, several functional magnetic resonance imaging (fMRI) studies have shown altered cortical activity in glaucoma patients (Qing et al., 2010; Gerente et al., 2015; Jiang et al., 2017; Wang et al., 2020). It is largely unknown how these anatomical and functional changes affect the integrity of cortical functional networks and how, in turn, changes in these networks affect visual functioning of patients. Understanding this may affect the applicability of future treatments; the modulation of brain activity could be integrated as an option for rehabilitation or training. Despite this, there have been very few studies assessing the local functional connectivity (FC) between specific regions of interest (ROI) in the brain of glaucoma participants. Moreover, in most studies, fMRI was applied while observers performed a task, which means that patients' vision impairment could have affected both performance and imaging results (Qing et al., 2010; Gerente et al., 2015). It has been shown that using resting state (RS) blood oxygenation level-dependent (BOLD) signals is an effective way in assessing neuronal activity, and specifically, brain functional changes (van den Heuvel and Hulshoff Pol, 2010). RS is a particularly attractive approach to study populations with neuro-ophthalmic diseases like glaucoma. Since the patients do not need to perform any task, it can be used even when they are severely visually impaired as their impairment at the level of the eye will not affect the results (Frezzotti et al.,
2014, 2016; Wang et al., 2016b, 2017; Giorgio et al., 2018; Carvalho et al., 2019). By examining which areas of the brain are activated synchronously during RS scans, it is possible to cluster certain areas into resting-state networks (RSN) (van den Heuvel and Hulshoff Pol, 2010). These results indicate that the brain consists of multiple cooperative systems or networks. The known RSNs are named based on their function and include, amongst others the visual network (VN), the salience network $(\mathrm{SN})$, dorsal attention network (DAN), and the default mode network (DMN). By examining FC within and between these predefined networks, it is possible to determine to what extent a pathology, such as glaucoma, affects the integrity of a participant's brain networks.

The first aim of our study is to determine network integrity in glaucoma. For this, we characterized functional network activity over time and identified the most central brain areas ("hubs") in glaucoma patients and control subjects. Fast eigenvector centrality mapping (fECM) was applied to each time course to attribute an eigenvector centrality (EC) value to each predefined region of interest (ROI). For each scan and for each group, the ROIs with EC values higher than the 95th percentile were considered the most central brain regions ("hubs"). Hubs for which we found a significant difference in EC between glaucoma and healthy in both scans were considered to provide evidence for network changes. Importantly, as mentioned before, by basing this analysis on resting-state (RS) fMRI data, we assess network integrity independently from the quality of the input coming via the eyes.

Our second aim is to investigate the different ways in which the VF could affect the hub function of networked brain areas. Therefore, in our study we defined three different behavioral scores based on VF sensitivity. These were calculated based on the notion that a brain region's hub function might relate to: (1) the sensitivity of the worse eye - indicating disease severity, calculated by selecting the mean deviation (MD) value of the worse eye (WorseMD); (2) the sensitivity of both eyes combined - with one eye potentially compensating for loss in the other, calculated by taking the best value from overlapping locations in the total deviation maps of left and right eye and then calculating the mean of these values (Binocular Integrated Visual Field - BIVF); or (3) a difference in eye sensitivity - requiring additional network interactions, calculated as the absolute difference between the MD values of the right and left eye (AbsDiffMD). Next, we calculated the correlation between these behavioral scores and the centrality of the brain regions identified as being most influential in either glaucoma or control participants. 


\section{MATERIALS AND METHODS}

\section{Study Population}

Twenty patients with primary open-angle glaucoma (POAG) and twenty-four control participants were included. Patients were invited from the glaucoma database (Heeg and Jansonius, 2009) of the University Medical Center Groningen (UMCG); healthy controls responded to advertisements distributed around the UMCG and the city of Groningen. Participants' demographics are detailed in Table 1. Patients and controls did not differ regarding age $(p=0.3)$. Participants were invited to the screening ophthalmic session only if they passed our exclusion criteria, i.e. had no past or current psychiatric disorder; no claustrophobia; had no MRI incompatible implants or non-MRI safe tattoos, and did not use recreational drugs or medications (which may influence neurodegenerative progression). Prior to the screening and experiment, participants signed an informed consent form. The study protocol was approved by the ethics board of the University Medical Center Groningen (UMCG). The study followed the tenets of the Declaration of Helsinki.

\section{Ophthalmic Data Collection}

All participants took part in an ophthalmic session, which included visual acuity (VA) examination, intraocular pressure (IOP) measurement, optical coherence tomography (OCT), and VF assessment using standard automated perimetry (SAP).

Glaucoma participants had, based on previous regular appointments not related to this study, been diagnosed with POAG in at least one of their eyes and have a SAP mean deviation (MD) of $-2 \mathrm{~dB}$ or lower, as measured with the Humphrey Visual Field Analyzer (HFA; Carl Zeiss Meditec, Dublin, CA, USA; SITA FAST strategy with either the 30-2 or 24-2 grid). Participants were included only if their VF loss was strictly due to glaucoma. They also had to have abnormal values in at least one eye for the thickness of the retinal nerve fiber layer (RNFL), as assessed by OCT (Canon HS-100, software version 4.1.0, Tokyo, Japan).

Healthy participants were required to have a best-corrected VA of at least 0.1 logMar ( 0.8 decimal $)$ in both eyes. Their IOP had to be below $21 \mathrm{mmHg}$, as assessed with a non-contact tonometer (Tonoref II, Nidek, Aichi, Japan). VF integrity was verified using the HFA, with the MD required to be above -2 $\mathrm{dB}$. Their OCT assessment had to show a normal thickness of the RNFL in both eyes (no clock hours below 1st percentile allowed).

\section{MRI and fMRI Data Acquisition}

Scanning was carried out on a 3 Tesla Siemens Prisma MRIscanner using a 64-channel receiving head coil. A T1-weighted scan (voxel size, $1 \mathrm{~mm}^{3}$; matrix size, $256 \times 256 \times 256$ ) covering the whole-brain was recorded to chart each participant's cortical anatomy. Padding was used for a balance between comfort and reduction of head motion. The functional scans were collected using standard EPI sequence with 260 volumes (TR, 1,350 ms; TE, $30 \mathrm{~ms}$; voxel size, $2 \times 2 \times 3 \mathrm{~mm}^{3}$, flip angle of $68^{\circ}$; matrix size, $94 \times 94 \times 45$ ). Two $\mathrm{fMRI}$ resting-state scans were taken in a room in complete darkness for each participant. During both resting-state functional scans, participants were asked to close their eyes, relax, not think about anything in particular, and to try not to move.

\section{fMRI Data Analyses}

Image pre-processing, FC, ECM and statistical analyses were performed using SPM12 (Wellcome Department of Imaging Neuroscience, London, UK), fastECM toolbox (Wink et al., 2012), MarsBaR Region of interest (ROI) toolbox (http:// marsbar.sourceforge.net/) and customized scripts, implemented in Matlab 2016b (The Mathworks Inc., Natick, Massachusetts). A related toolbox containing the code will be made available via the website www.visualneuroscience.nl.

\section{Image Preprocessing}

For each subject, the structural magnetic resonance image was co-registered and normalized against the Montreal Neurological Institute (MNI) template and segmented in order to obtain white matter (WM), gray matter (GM) and cerebrospinal fluid (CSF) probability maps in the MNI space. FMRI data were spatially realigned, co-registered to the MNI-152 EPI template and subsequently normalized utilizing the segmentation option for EPI images in SPM. All normalized data were denoised

TABLE 1 | Demographic and clinical information of participants.

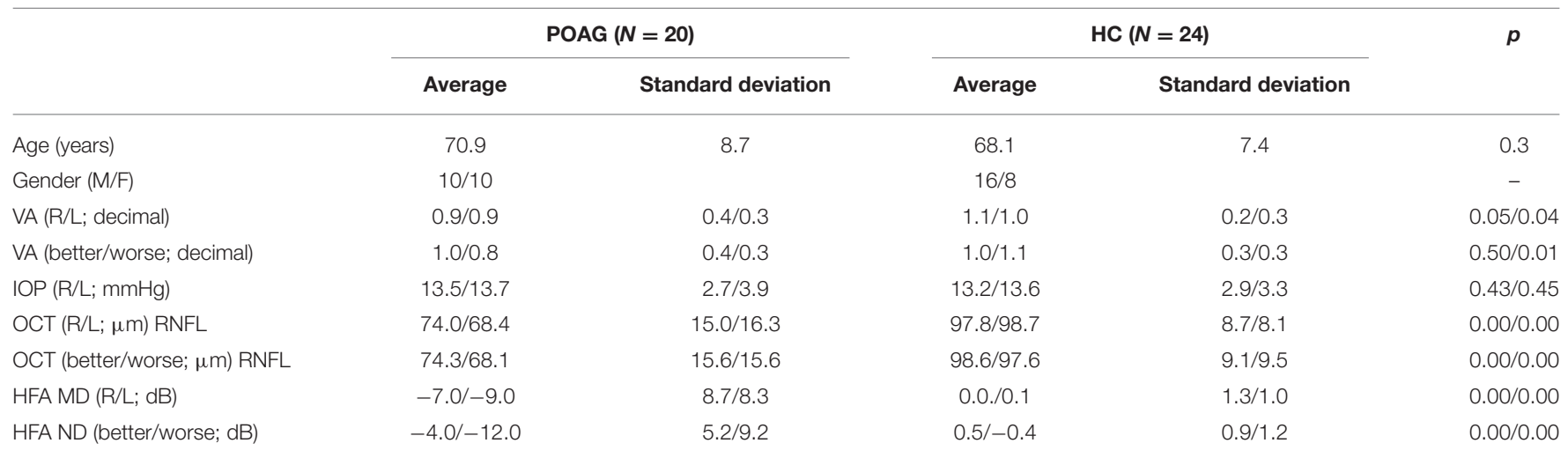

POAG, primary open-angle glaucoma; HC, healthy controls; VA, visual acuity; IOP, intraocular pressure (with treatment for glaucoma); OCT RNFL, optical coherence tomography, retinal nerve fiber layer thickness; HFA MD, humphrey visual field analyzer, mean deviation; better/worse, eye selected based on better and worse HFA MD. 
using ICA-AROMA (Pruim et al., 2015). Additionally, spatial smoothing was applied (8 millimeters) to the fMRI data. No global signal regression was applied.

Based on the Power atlas coordinates (Power et al., 2011), 11 functional networks and associated 232 regions of interest (ROI) were defined ( $5 \mathrm{~mm}$ radius) using the MarsBar ROI toolbox for SPM12 (Brett et al., 2002, n.d.). For each ROI, a time-series was extracted by averaging across voxels per time point. Due to their related functions, some of the networks were excluded or modified: the sensory/somatomotor hand and mouth networks were combined into one network, the cerebellar network was excluded since our analysis focused more on cortical structures and the uncertain network was completely excluded from the analysis due to interpretation difficulties - due to the nature of uncertain networks, it is unknown what function each specific ROI pertains to. This left 131 ROIs spread over seven networks: DMN, memory network, visual network, salience network, ventral attention network and dorsal attention network.

\section{Prewhitening}

To facilitate statistical inference, data were "pre-whitened" by removing the estimated autocorrelation structure in a two-step GLM procedure (Monti, 2011; Bright and Murphy, 2015). In the first step, the raw data were filtered against the 6 motion parameters ( 3 translations and 3 rotations). Using the resulting residuals, the autocorrelation structures present in the data were estimated using an Auto-Regressive model of order 1 [AR(1)] and then removed from the raw data. Next, the realignment parameters, white matter (WM) and cerebrospinal fluid (CSF) signals were removed as confounders on the whitened data.

\section{Functional Connectivity Analysis}

For each participant, the pairwise temporal Pearson correlation between ROIs was calculated and a Fisher's z-transformation was applied. The ROI's z-values (hereafter: FC values) were averaged across participants. Then, the median group FC-values were used for the whole-brain analysis and used to compare the FCvalues between groups using a family-wise error corrected (FWE) permutation test. Permuting the participant's group labels was repeated 10,000 times per participant, $p \leq 0.05$ was considered statistically significant.

\section{Eigenvector Centrality Analysis}

To determine the most important hubs of the predefined networks, fast ECM (Wink et al., 2012) was performed on the defined ROI time course data per subject. The ECM method builds on the concept of node centrality, which characterizes functional networks active over time and attributes a voxel-wise centrality value to each ROI. Such a value is strictly dependent on the sum of centrality properties of the direct neighbor nodes within a functional network. In the fast ECM toolbox (Wink et al., 2012), ECM is estimated from the adjacency matrix, which contains the pairwise correlation between the ROIs. To obtain a real-valued EC value, we added +1 to the values in the adjacency matrix. Several EC values can be attributed to a given node, but only the eigenvector with the highest eigenvalue (EV) will be used for further analyses. The highest EV values were averaged across subjects at group level. Based on these values, influential ROIs, i.e. the hubs (van den Heuvel and Sporns, 2011; Mišić et al., 2015; Betzel et al., 2016), can then be identified. Per group, only ROIs with EC coefficients higher than the 95th percentile (5\% highest) were considered the most central and therefore used in the following analyses. Based on literature, the 95th percentile was chosen as an arbitrary threshold (Invernizzi et al., 2019). Furthermore, we quantify possible differences between influential hubs across groups by permuting labels with 10,000 time repetitions. ROIs with $p \leq 0.05$ were considered statistically significant and therefore, categorized as well as influential hubs. FWE correction was applied for the number of group level comparisons, but not for the total number of ROIs analyzed. Only FWE corrected $p$-values are reported.

In addition, a proxy distribution for the null hypothesis ( $\mathrm{H} 0)$ was obtained by generating 1,000 times surrogate BOLD time

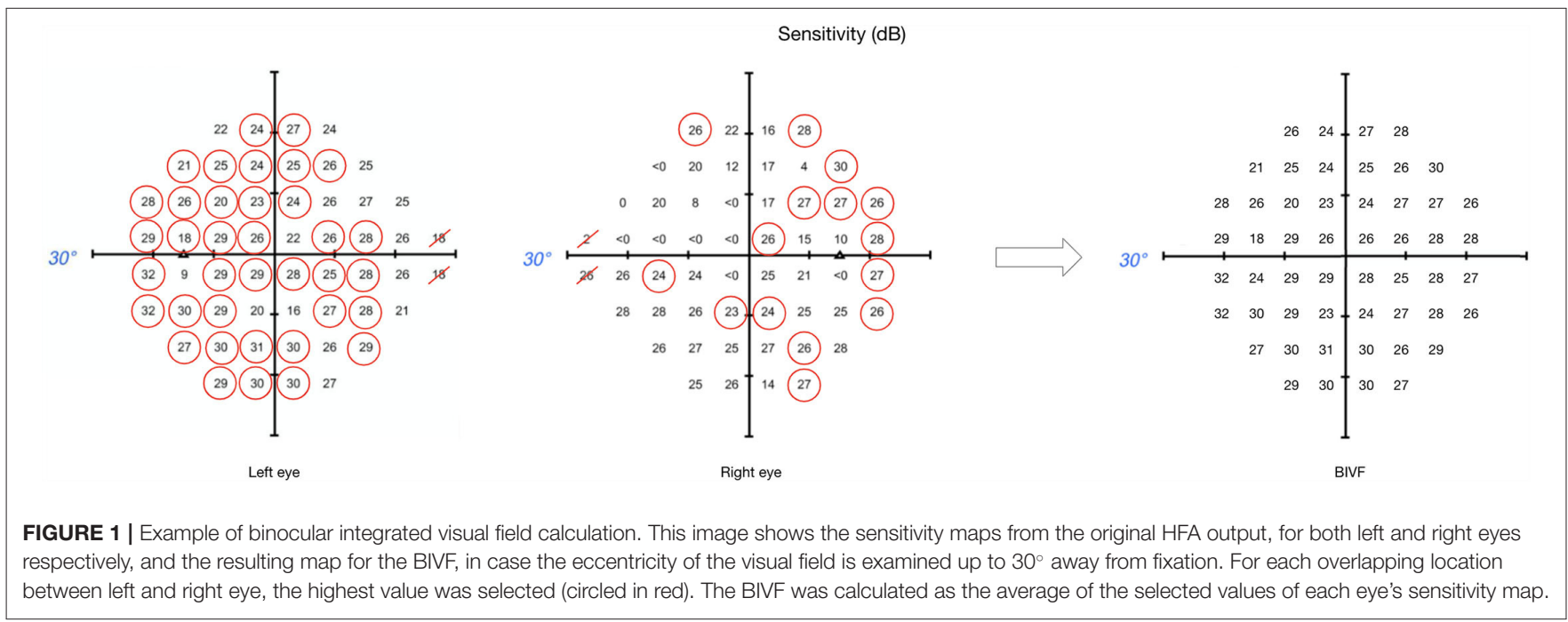


series using the iterative amplitude adjusted Fourier transform method (iAAFT; Schreiber and Schmitz, 1996; Räth and Monetti, 2009). In this way, correlations between ROIs were removed; the null distribution represents the amount of centrality obtained when no functional communication is present in the brain. Note that the null distribution of the ECM is not centered at zero, as EC values are forced to be positive real-valued. To define the confidence intervals of each EC value estimated per ROI, a bootstrap technique (across time-point) was used at group level in parallel to resample the filtered fMRI data. To support visualization, a Gaussian distribution was fitted to both bootstrap and surrogate distributions.

\section{Behavioral Scores}

The behavioral scores were derived from the HFA original output by: (1) selecting the mean deviation (MD) value of the worse eye (WorseMD); (2) taking the highest value from overlapping locations in the total deviation maps of the left and the right eye and then calculating the mean of these values (Figure 1; Binocular Integrated Visual Field - BIVF) (Crabb and Viswanathan, 2005); (3) calculating the absolute difference between the MD values of the right and left eye (AbsDiffMD). Due to fatigue, 17 of the 24 healthy controls participants and 19 of the 20 participants with glaucoma completed the study-related visual field assessment that enabled us to calculate these scores.

\section{Hub Selection for Correlation Analysis}

For the correlation analysis of EC values to the behavioral scores, our selection of ROIs was done as follows. ROIs were included that were identified as hub based on the data of the glaucoma participants (RS1 or RS2) or the data of the control participants (RS1 or RS2). In other words, for this analysis, the ROIs were selected only based on them fulfilling the hub criterion per scan and not on a difference between the control and glaucoma participants.

In the analysis, each behavioral score of a participant (Section Image Preprocessing) was correlated with their EC value per
ROI (separately for each scan) using Spearman's correlation. A correlation of $p \leq 0.05$ was considered significant.

\section{RESULTS}

\section{Whole Brain Connectivity Analysis}

To compare whole brain connectivity between control and glaucoma participants, we plotted the averaged FC scores across all ROIs (Figure 2A) and the average EC values per ROI in both groups (Figures 2B,C, for controls and glaucoma participants, respectively). No significant differences were found with any of these analyses (RS1: HC vs. POAG, $p=0.59$; RS2: HC vs. POAG, $p=0.089$ ).

Moreover, no significant differences were found with either the whole-brain-within or -between functional connectivity network analyses for controls and glaucoma groups (for further analyses see the Supplementary Figures S1-S3).

\section{Identification of Influential Brain Areas}

Based on the EC values averaged across participants, we identified the $5 \%$ most central ROIs per group, which we refer to as "hubs" (Figure 3). For controls, hubs belonged to the Visual, Salience, Ventral and Dorsal Attention networks. For POAG, hubs belonged to the Visual, Salience, Default Mode, Ventral and Dorsal Attention networks. Based on the first RS scan, differences in EC between the POAG and controls did not reach significance for any of these hubs. In the second RS scan, differences reached significance for two hubs, both belonging to the Salience Network (Insula, $p=0.023$; Frontal Middle Gyrus, $p$ $=0.016$ - Supplementary Figure S5).

To further explore and quantify the difference in EC value between the healthy and glaucoma participants, bootstrap and surrogate methods were applied to the EC values of the hubs identified in both RS scans. Figure 4 shows the bootstrapped and the surrogate EC distributions for a number of hubs present in both RS scans for healthy and glaucoma groups (Figures 4A,B). Furthermore, we are reporting in the Supplementary Material the EC distributions for the two hubs for which differences
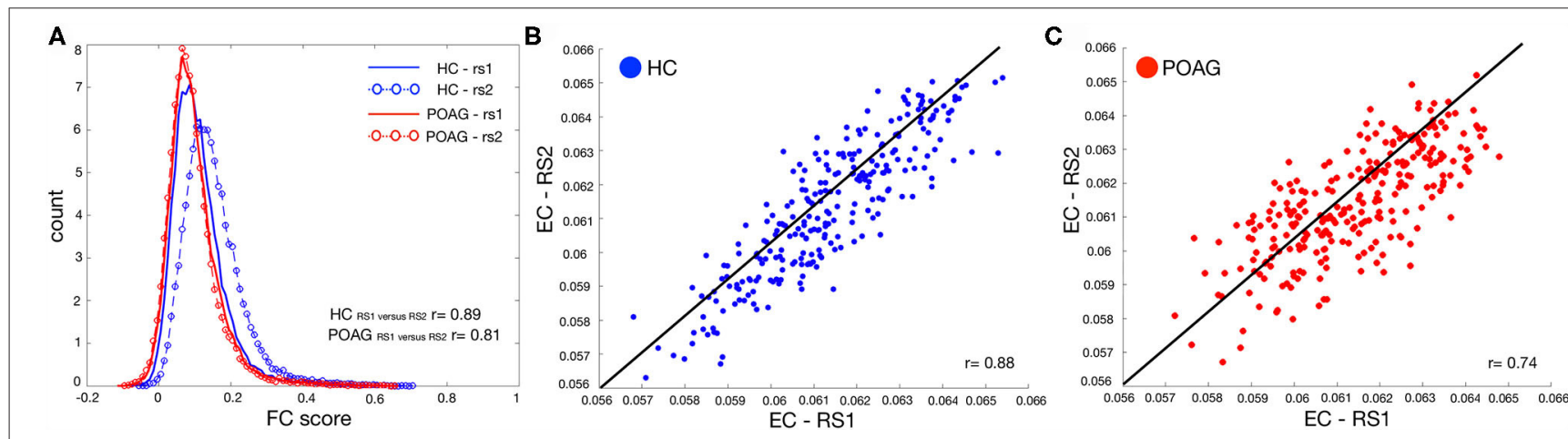

FIGURE 2 | Test-retest evaluation between RS scans for both FC and EC values. Panel (A) shows the histogram of the functional connectivity (FC) scores across all ROls computed for both RS scans for control (blue line and circles) and POAG (red line and circles) participants. The y-axis label "count" represents the number of ROls with a particular functional connectivity value. Panels $\mathbf{( B , C )}$ report the average EC values for control (blue) and POAG (red) groups, respectively. Each dot represents the average EC value for a single ROI defined based on the Power atlas. The black line represents the linear fit applied to the data. 

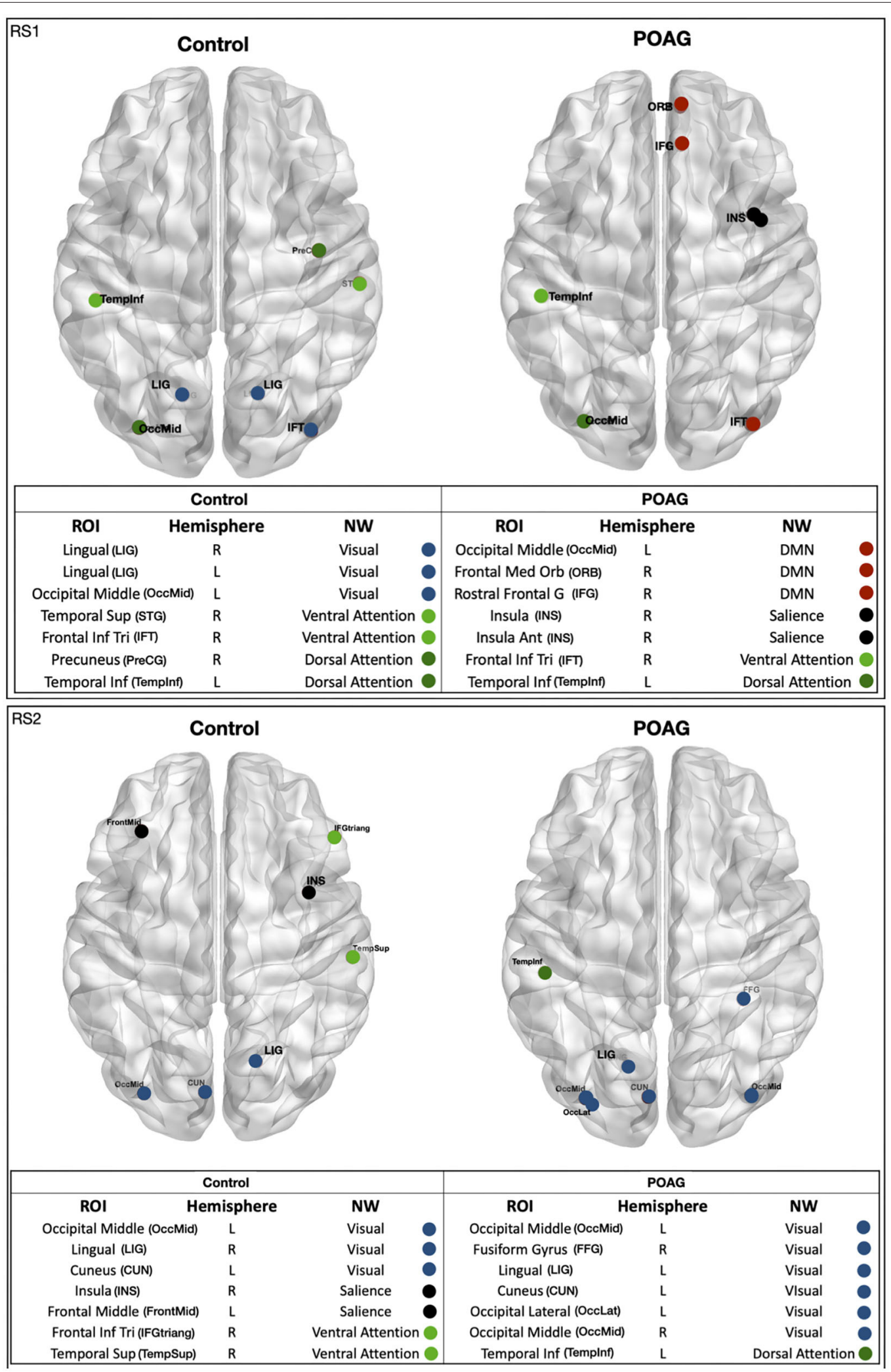

FIGURE 3 | Functional network hubs identified in either of the two RS scans. The figure shows all identified hubs, i.e. the ROls with $5 \%$ highest eigenvector centrality in either of the RS scans in either group - healthy and POAG participants. The tables below the figures list the full name of the ROI, the ROI's hemisphere, the name 
FIGURE 3 I of the ROI's functional NW and the NW color code. ROI, region of interest; CUN, cuneus; IFT, frontal inferior triangularis; ORB, frontal medial orbital; FrontMid, frontal middle; FFG, fusiform gyrus; INS, insula; INS, insula anterior; LIG, lingual gyrus; OccLat, occipital lateral; OccMid, occipital middle; PreCG, precuneus; IFG/IFGtriang, rostral frontal gyrus; Templnf, temporal inferior; TempSup, temporal superior; STG, temporal superior gyrus. Hemisphere: R, right; L, left. NW, network; DMN, default mode network; Salience, dorsal attention; Visual, ventral attention.

reached significance in RS2 (Insula and Frontal Middle Gyrus; Supplementary Figure S5).

\section{Correlations of EC of Hubs With Behavioral Scores}

The median behavioral scores were as follows. The Worse MD score for the participants with glaucoma was -12.11 [IQR 13.60] $\mathrm{dB}$, while for controls it was -0.31 [IQR 0.82] $\mathrm{dB}$. The BIVF score for the participants with glaucoma was -2.63 [IQR 3.45] $\mathrm{dB}$, while for controls it was 0.79 [IQR 0.37] $\mathrm{dB}$. The AbsDiffMD score for the participants with glaucoma was 4.82 [IQR 6.65] dB while for controls it was 0.75 [IQR 0.59] dB. For all identified hubs, based on the data of the first RS scan we found significant correlations between the EC values of the right Lingual Gyrus (LIG) and the BIVF $(p=0.04)$, the AbsDiffMD $(p=0.03)$, and the WorseMD ( $p=0.04)$ and (Figure 5, top panels). Based on the data of the second RS scan, of these correlations, the one between the right Lingual Gyrus (LIG) and the BIVF reached significance again (BIVF $p=0.05$; AbsDiffMD $p=0.69$; WorseMD $p=0.23$; Figure 5, bottom panels). In other words, only the correlation between the right LIG and BIVF resulted in a reproducible finding. The right LIG belongs to the Visual Network (Power et al., 2011). In the Supplementary Materials, we report the bootstrapped and surrogate distributions for the hubs that showed no significant correlation between the EC values and the behavioral scores are shown (Supplementary Figure S4).

\section{DISCUSSION}

This study has two main findings, one at the global and one at the local brain level. First, in glaucoma, global brain network communication is preserved. We conclude this based on an absence of whole brain differences in functional connectivity between glaucoma participants and controls. Secondly, in glaucoma, local brain hub connectivity relates to functional visual abilities. We conclude this because the hub function of the Lingual Gyrus is associated with the sensitivity of the integrated VF of both eyes. Below, we discuss these conclusions in more detail as well as the implications for understanding cortical functioning of glaucoma patients.

\section{Global Brain Network Communication Is Preserved in Glaucoma}

No functional connectivity disruptions were found either withinor between- functional networks of glaucoma participants and controls. This is consistent with a previous study (Wang et al., 2016b). Since global disruption of functional connectivity has been found in other syndromes such as Alzheimer's disease and Parkinson's disease (Pievani et al., 2011), these results support the idea that glaucoma has a different neuropathogenesis. Several other studies found functional changes at the level of single brain areas in glaucoma, however some of these studies investigated just a few specific vision-related brain areas, or focused only on FC within a specific set of networks, not between different networks (Dai et al., 2013; Frezzotti et al., 2014; Li et al., 2014; Song et al., 2014; Wang et al., 2017; Giorgio et al., 2018; Trivedi et al., 2019; Qu et al., 2020).

In our study, we found that, based on one of our RS scans, the centrality of the insula and the frontal middle gyrus, both part of the Salience Network, differed between glaucoma participants and controls. Yet, this finding did not replicate in the other scan. Overall, we conclude that the evidence for changed FC or centrality in glaucoma is weak at best.

\section{Reduced Centrality of the Lingual Gyrus Is Associated With Reduced Functional Vision}

Based on a set criterion for their EC value, we identified a number of brain areas as being hubs. For these hubs, we found that the centrality of the right LIG related consistently to the sensitivity of the binocular integrated VF (BIVF) of participants. While the EC of a few other hubs (Insula and Frontal Middle Gyrus) showed a correlation with one of the behavioral VF indices in either one of the scans, only the correlation of the EC of the right LIG with the sensitivity of the binocular integrated VF (BIVF) reproduced in both scans. We can understand this result based on two previous findings. First, the LIG is known for its role in visuospatial processing and topographical recognition. It was found that lesions in this area affect the ability of patients to orient themselves (Takahashi and Kawamura, 2002; Mendez and Cherrier, 2003). Secondly, glaucoma patients often report issues in orientating and moving in their surroundings. Previously, this self-perceived visual disability was shown to relate most strongly to peripheral vision loss when expressed in terms of the BIVF score (Crabb and Viswanathan, 2005). This implies the centrality of the LIG directly relates to this functional visual ability. It remains to be determined whether this relationship also implies local reorganization of the LIG. The reduced centrality of the LIG may either be a consequence of patients' reduced visual input, mediated by their reduced orientation and mobility performance or a consequence of both. Irrespective, the fact that we consistently find this relationship based on RS data acquired in the absence of visual stimulation implies this altered centrality of the LIG has a permanent basis, implying brain reorganization has occurred.

\section{Clinical Implications}

The results in this study imply that network functionality poses no limit to future treatment options. The central role of the LIG suggests that future therapies, such as brain stimulation, could 


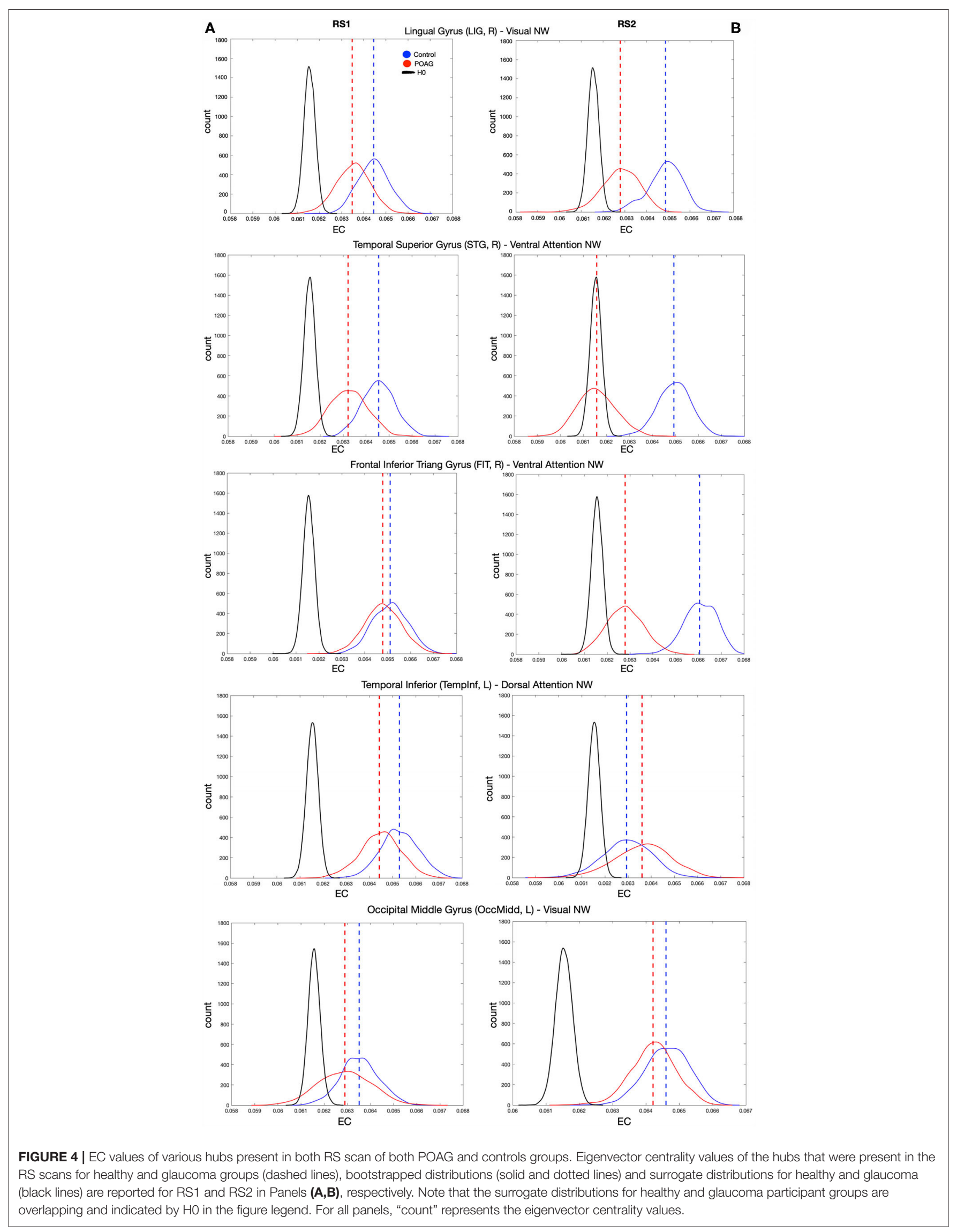




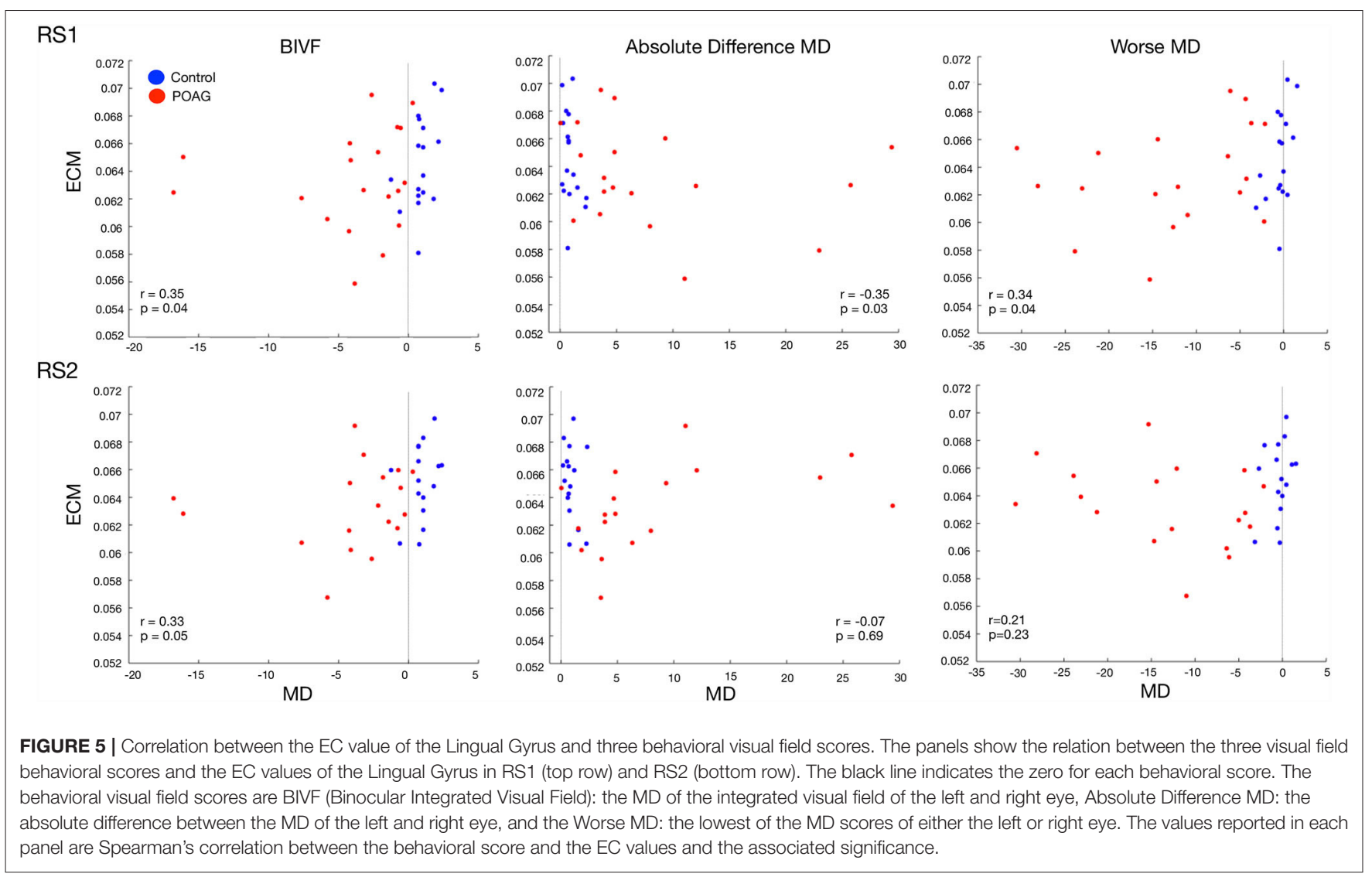

focus on this brain area, as its modulation may benefit glaucoma patients (Sabel et al., 2020). Moreover, the notion that orientation and mobility performance are also part of this equation could support the introduction of cognition-based mobility training in the treatment (Virgili and Rubin, 2010; Gunn et al., 2019).

\section{Limitations and Future Studies}

In this study, due to our relatively small sample size, we could not meaningfully divide the group in smaller subgroups. Consequently, in our glaucoma group, some participants had early or mild glaucoma and VFs that were mostly intact, while others had severe glaucoma and visual losses that caused a near complete blindness in one eye. This spread in patient characteristics was actually beneficial for our ability to relate centrality to functional vision. However, it makes it harder to detect more subtle patterns of reorganization. Future studies on larger samples of patients might address the presence of subtle functional connectivity changes at different glaucoma stages. For example, it is known that in early POAG, the brain goes through a stage where brain structures become larger before they become atrophic (Williams et al., 2013). This would result in compensatory changes in which neural activity is initially suppressed and then enhanced at a later stage of the disease (Johansen-Berg, 2007). Besides measuring the visual fields, such studies should also consider integrally assessing or querying patients on their mobility. Since our present crosssectional study cannot, future longitudinal studies in glaucoma
(Haykal et al., 2021) could help to establish plausible causal relationships between behavioral and brain changes.

\section{CONCLUSION}

We found no consistent alterations in the global or local functional networks of glaucoma participants. However, our study did show that the integrity of the hub function of the LIG relates to patients' functional vision as expressed in their BIVF score. Future studies are required to determine whether deficits experienced by glaucoma patients in orientation and mobility are related to this observation, and if so, whether they could be either a consequence or a cause of changes in this brain area.

\section{DATA AVAILABILITY STATEMENT}

The raw data supporting the conclusions of this article will be made available by the authors, without undue reservation.

\section{ETHICS STATEMENT}

The studies involving human participants were reviewed and approved by Ethics board of the University Medical Center Groningen (UMCG). The patients/participants provided their written informed consent to participate in this study. 


\section{AUTHOR CONTRIBUTIONS}

$\mathrm{GD}, \mathrm{AI}$, and FC contributed to conception and design of the study. AI, JC, and DO performed data collection. AI and GD organized the database. AI performed the statistical analysis. FC and RR supervised the work. GD and AI wrote the first draft of the manuscript. All authors contributed to manuscript revision, read, and approved the submitted version.

\section{FUNDING}

FC, AI, GD, and JC received funding from the European Union's Horizon 2020 research and innovation program under the Marie Sklodowska-Curie grant agreement No. 661883/675033/641805 (EGRET cofund, EGRET+ and NextGenVis). AI, GD, and JC

\section{REFERENCES}

Betzel, R. F., Gu, S., Medaglia, J. D., Pasqualetti, F., and Bassett, D. S. (2016). Optimally controlling the human connectome: the role of network topology. Sci. Rep. 6, 30770. doi: 10.1038/srep30770

Brett, M., Anton, J.-L., Valabregue, R., and Poline, J.-B. (2002). Region of interest analysis using an SPM toolbox. [Abstract]. Presented at the 8th International Conference on Functional Mapping of the Human Brain, June 2-6, Sendai, Japan - ScienceOpen. Available online at: https://www.scienceopen.com/ document?vid=f9a14c27-7c57-4539-933d-d73833ff5232 (accessed May 13, 2020)

Bright, M. G., and Murphy, K. (2015). Is fMRI “noise” really noise? Resting state nuisance regressors remove variance with network structure. NeuroImage. 114, 158-169. doi: 10.1016/j.neuroimage.2015.03.070

Carvalho, J., Renken, R. J., and Cornelissen, F. W. (2019). Studying cortical plasticity in ophthalmic and neurological disorders: from stimulusdriven to cortical circuitry modeling approaches. Neural Plastic. 2724101. doi: 10.1155/2019/2724101

Chen, Z., Lin, F., Wang, J., Li, Z., Dai, H., Mu, K., et al. (2013). Diffusion tensor magnetic resonance imaging reveals visual pathway damage that correlates with clinical severity in glaucoma. Clin. Exper. Ophthalmol. 41, 43-49. doi: 10.1111/j.1442-9071.2012.02832.x

Crabb, D. P., and Viswanathan, A. C. (2005). Integrated visual fields: a new approach to measuring the binocular field of view and visual disability. Graefe's Arch. Clin. Experi. Ophthalmol. 243, 210-216. doi: 10.1007/s00417-004-0984-x

Dai, H., Morelli, J. N., Ai, F., Yin, D., Hu, C., Xu, D., et al. (2013). Restingstate functional MRI: functional connectivity analysis of the visual cortex in primary open-angle glaucoma patients. Human Brain Mapp. 34, 2455-2463. doi: 10.1002/hbm.22079

Frezzotti, P., Giorgio, A., Motolese, I., De Leucio, A., Iester, M., Motolese, E., et al. (2014). Structural and functional brain changes beyond visual system in patients with advanced glaucoma. PLoS ONE. 9, e105931. doi: 10.1371/journal.pone.0105931

Frezzotti, P., Giorgio, A., Toto, F., De Leucio, A., and De Stefano, N. (2016). Early changes of brain connectivity in primary open angle glaucoma. Human Brain Mapp. 37, 4581-4596. doi: 10.1002/hbm.23330

Gerente, V. M., Schor, R. R., Chaim, K. T., Felix, M., de, M., Ventura, D. F., et al. (2015). Evaluation of glaucomatous damage via functional magnetic resonance imaging, and correlations thereof with anatomical and psychophysical ocular findings. PLoS ONE. 10, e0126362. doi: 10.1371/journal.pone.0126362

Giorgio, A., Zhang, J., Costantino, F., De Stefano, N., and Frezzotti, P. (2018). Diffuse brain damage in normal tension glaucoma. Human Brain Mapp. 39, 532-541. doi: 10.1002/hbm.23862

Gunn, S. M., Lajoie, K., Zebehazy, K. T., Strath, R. A., Neima, D. R., and Marigold, D. S. (2019). Mobility-related gaze training in individuals with glaucoma: a proof-of-concept study. Transl. Vis. Sci. Technol. 8, 23-23. doi: $10.1167 /$ tvst.8.5.23 received additional funding from the Graduate School of Medical Sciences (GSMS), University of Groningen, The Netherlands. The funding organizations had no role in the design, conduct, analysis, or publication of this research.

\section{ACKNOWLEDGMENTS}

We would like thank Prof. Dr. Nomdo Jansonius for his help in verifying the quality of the ophthalmic patient data.

\section{SUPPLEMENTARY MATERIAL}

The Supplementary Material for this article can be found online at: https://www.frontiersin.org/articles/10.3389/fnagi. 2021.744139/full\#supplementary-material

Gupta, N., and Yücel, Y. H. (2007). What changes can we expect in the brain of glaucoma patients? Surv. Ophthalmol. 52, S122-S126. doi: 10.1016/j.survophthal.2007.08.006

Haykal, S., Jansonius, N. M., and Cornelissen, F. W. (2021). Progression of visual pathway degeneration in primary open-angle glaucoma: a longitudinal study. Front. Hum. Neurosci. 15, 630898. doi: 10.3389/fnhum.2021.630898

Heeg, G., Jansonius, N. (2009). The groningen longitudinal glaucoma study III. The predictive value of frequency-doubling perimetry and GDx nerve fibre analyser test results for the development of glaucomatous visual field loss. Eye. 23, 1647-1652. doi: 10.1038/eye.2008.348

Hernowo, A. T. (2012). Visual pathway morphometry in visual field defects. Available online at: http://irs.ub.rug.nl/ppn/340376880?utm_source= rssandutm_medium=rssandutm_campaign=visual-pathway- morphometryin-visual-field-defects (accessed April 1, 2021).

Invernizzi, A., Halbertsma, H. N., van Ackooij, M., Bais, L., Boertien, J., Renken, R. J., et al. (2019). rTMS treatment of visual hallucinations using a connectivity-based targeting method: a case study. Brain Stimul. 12, 1622-1624. doi: 10.1016/j.brs.2019.09.003

Jiang, M.-M., Zhou, Q., Liu, X.-Y., Shi, C.-Z., Chen, J., and Huang, X.-H. (2017). Structural and functional brain changes in early- and mid-stage primary openangle glaucoma using voxel-based morphometry and functional magnetic resonance imaging. Medicine. 96, e6139. doi: 10.1097/MD.0000000000006139

Johansen-Berg, H. (2007). Structural plasticity: rewiring the brain. Curr. Biol. 17, R141-R144. doi: 10.1016/j.cub.2006.12.022

Li, T., Liu, Z., Li, J., Liu, Z., Tang, Z., Xie, X., et al. (2014). Altered amplitude of low-frequency fluctuation in primary open-angle glaucoma: a resting-state FMRI study. Investigat. Ophthalmol. Visual Sci. 56, 322-329. doi: 10.1167/iovs.14-14974

Mendez, M. F., and Cherrier, M. M. (2003). Agnosia for scenes in topographagnosia. Neuropsychologia. 41, 1387-1395. doi: 10.1016/S0028-3932(03)00041-1

Mišić, B., Betzel, R. F., Nematzadeh, A., Goñi, J., Griffa, A., Hagmann, P., et al. (2015). Cooperative and competitive spreading dynamics on the human connectome. Neuron. 86, 1518-1529. doi: 10.1016/j.neuron.2015.05.035

Monti, M. M. (2011). Statistical analysis of fMRI time-series: a critical review of the GLM approach. Front. Hum. Neurosci. 5, 28. doi: 10.3389/fnhum.2011.00028

Nucci, C., Martucci, A., Cesareo, M., Mancino, R., Russo, R., Bagetta, G., et al. (2013). Brain involvement in glaucoma: advanced neuroimaging for understanding and monitoring a new target for therapy. Curr. Opin. Pharmacol. 13, 128-133. doi: 10.1016/j.coph.2012. 08.004

Pievani, M., de Haan, W., Wu, T., Seeley, W. W., and Frisoni, G. B. (2011). Functional network disruption in the degenerative dementias. Lancet Neurol. 10, 829-843. doi: 10.1016/S1474-4422(11)70158-2

Power, J. D., Cohen, A. L., Nelson, S. M., Wig, G. S., Barnes, K. A., Church, J. A., et al. (2011). Functional network organization of the human brain. Neuron. 72, 665-678. doi: 10.1016/j.neuron.2011.09.006 
Pruim, R. H. R., Mennes, M., van Rooij, D., Llera, A., Buitelaar, J. K., and Beckmann, C. F. (2015). ICA-AROMA: A robust ICA-based strategy for removing motion artifacts from fMRI data. NeuroImage. 112, 267-277. doi: 10.1016/j.neuroimage.2015.02.064

Qing, G., Zhang, S., Wang, B., and Wang, N. (2010). Functional MRI signal changes in primary visual cortex corresponding to the central normal visual field of patients with primary open-angle glaucoma. Investigat. Ophthalmol. Visual Sci. 51, 4627-4634. doi: 10.1167/iovs.09-4834

Qu, H., Wang, Y., Yan, T., Zhou, J., Lu, W., and Qiu, J. (2020). Data-driven parcellation approaches based on functional connectivity of visual cortices in primary open-angle glaucoma. Investigat. Ophthalmol. Visual Sci. 61, 33. doi: $10.1167 /$ iovs.61.8.33

Quigley, H. A., and Broman, A. T. (2006). The number of people with glaucoma worldwide in 2010 and 2020. Br. J. Ophthalmol. 90, 262-267. doi: 10.1136/bjo.2005.081224

Räth, C., and Monetti, R. (2009). Surrogates with Random Fourier Phases. In: Topics on Chaotic Systems. doi: 10.1142/9789814271349_0031

Sabel, B. A., Thut, G., Haueisen, J., Henrich-Noack, P., Herrmann, C. S., Hunold, A., et al. (2020). Vision modulation, plasticity and restoration using noninvasive brain stimulation: an IFCN-sponsored review. Clin. Neurophysiol. 131, 887-911. doi: 10.1016/j.clinph.2020.01.008

Schreiber, T., and Schmitz, A. (1996). Improved surrogate data for nonlinearity tests. Phys. Rev. Lett. 77, 635-638. doi: 10.1103/PhysRevLett.77.635

Song, Y., Mu, K., Wang, J., Lin, F., Chen, Z., Yan, X., et al. (2014). Altered spontaneous brain activity in primary open angle glaucoma: a restingstate functional magnetic resonance imaging study. PLOS ONE. 9, e89493. doi: 10.1371/journal.pone.0089493

Takahashi, N., and Kawamura, M. (2002). Pure topographical disorientation - the anatomical basis of landmark agnosia. Cortex; a J. Devoted Study Nerv. Syst Behav. 38, 717-725. doi: 10.1016/S0010-9452(08)70039-X

Tham, Y.-C., Li, X., Wong, T. Y., Quigley, H. A., Aung, T., and Cheng, C.-Y. (2014). Global Prevalence of Glaucoma and Projections of Glaucoma Burden through 2040. Ophthalmology. 121, 2081-2082090. doi: 10.1016/j.ophtha.2014.05.013

Trivedi, V., Bang, J. W., Parra, C., Colbert, M. K., O'Connell, C., Arshad, A., et al. (2019). Widespread brain reorganization perturbs visuomotor coordination in early glaucoma. Sci. Rep. 9, 14168. doi: 10.1038/s41598-019-50793-x

van den Heuvel, M. P., and Hulshoff Pol, H. E. (2010). Exploring the brain network: a review on resting-state fMRI functional connectivity. Eur. Neuropsychopharmacol. 20, 519-534. doi: 10.1016/j.euroneuro.2010.03.008

van den Heuvel, M. P., and Sporns, O. (2011). Rich-club organization of the human connectome. J. Neurosci. 31, 15775-15786. doi: 10.1523/JNEUROSCI.3539-11.2011

Virgili, G., and Rubin, G. (2010). Orientation and mobility training for adults with low vision. Cochrane Datab Syst Rev. 5, CD003925. doi: 10.1002/14651858.CD003925.pub3
Wang, J., Li, T., Sabel, B. A., Chen, Z., Wen, H., Li, J., et al. (2016a). Structural brain alterations in primary open angle glaucoma: a 3T MRI study. Sci. Rep. 6, 18969. doi: 10.1038/srep18969

Wang, J., Li, T., Wang, N., Xian, J., and He, H. (2016b). Graph theoretical analysis reveals the reorganization of the brain network pattern in primary open angle glaucoma patients. Eur. Radiol. 26, 3957-3967. doi: 10.1007/s00330-0164221- $\mathrm{x}$

Wang, J., Li, T., Zhou, P., Wang, N., Xian, J., and He, H. (2017). Altered functional connectivity within and between the default model network and the visual network in primary open-angle glaucoma: a resting-state fMRI study. Brain Imag. Behav. 11, 1154-1163. doi: 10.1007/s11682-0169597-3

Wang, Y., Lu, W., Xie, Y., Zhou, J., Yan, T., Han, W., et al. (2020). Functional alterations in resting-state visual networks in high-tension glaucoma: an independent component analysis. Front. Hum. Neurosci. 14, 330. doi: 10.3389/fnhum.2020.00330

Williams, A. L., Lackey, J., Wizov, S. S., Chia, T. M. T., Gatla, S., Moster, M. L., et al. (2013). Evidence for widespread structural brain changes in glaucoma: a preliminary voxel-based MRI study. Investigat. Ophthalmol. Vis. Sci. 54, 5880-5887. doi: 10.1167/iovs.13-11776

Wink, A. M., de Munck, J. C., van der Werf, Y. D., van den Heuvel, O. A., and Barkhof, F. (2012). Fast eigenvector centrality mapping of voxel-wise connectivity in functional magnetic resonance imaging: implementation, validation, and interpretation. Brain Connect. 2, 265-274. doi: 10.1089/brain.2012. 0087

Conflict of Interest: The authors declare that the research was conducted in the absence of any commercial or financial relationships that could be construed as a potential conflict of interest.

Publisher's Note: All claims expressed in this article are solely those of the authors and do not necessarily represent those of their affiliated organizations, or those of the publisher, the editors and the reviewers. Any product that may be evaluated in this article, or claim that may be made by its manufacturer, is not guaranteed or endorsed by the publisher.

Copyright (C) 2022 Demaria, Invernizzi, Ombelet, Carvalho, Renken and Cornelissen. This is an open-access article distributed under the terms of the Creative Commons Attribution License (CC BY). The use, distribution or reproduction in other forums is permitted, provided the original author(s) and the copyright owner(s) are credited and that the original publication in this journal is cited, in accordance with accepted academic practice. No use, distribution or reproduction is permitted which does not comply with these terms. 